http://jmscr.igmpublication.org/home/

ISSN (e)-2347-176x ISSN (p) 2455-0450

crossref DOI: https://dx.doi.org/10.18535/jmscr/v7i7.14

\title{
Demographic and Risk Factors Associated with Non-Tuberculous Mycobacteria from Tuberculosis Patients in Kaduna State, Nigeria
}

\author{
Authors \\ I. Ahmadu ${ }^{1,2}$, O.S. Olonitola ${ }^{1}$, A.B. Suleiman ${ }^{1}$ and D. Makolo ${ }^{3}$ \\ ${ }^{1}$ Department of Microbiology, Faculty of Life Sciences, Ahmadu Bello University, Zaria, Nigeria \\ ${ }^{2}$ National Tuberculosis Reference Laboratory, Zaria, Nigeria \\ ${ }^{3}$ Department of Sciences, School of Preliminary Studies, Kogi State Polytechnic, Lokoja, Nigeria
}

\begin{abstract}
Background: Nigeria is being ranked among the top five countries for tuberculosis (TB), Multi Drug Resistance TB and HIV deaths worldwide with the prevalence of $219 / 100,000$ population, $2.9 \%$ and $1.4 \%$ respectively. Non tuberculous mycobacteria (NTM) is an emerging health problem and an important cause of tuberculosis-like pulmonary disease. This study investigated the demographic and risk factors associated with Non tuberculous mycobacteria in Kaduna State, Nigeria.

Method: In a cross sectional study, structured questionnaire were used to collect demographic characteristics (age, sex, ethnicity, education, and marital status), health/behavioral factors (HIV, diabetes, smoking, alcohol use) and environmental exposures (farming, animal contact, and dust season).The questionnaires were administered at the point of sample collection by oral interview of the participants. The data generated were analyzed using Statistical Package for Social Sciences (SPSS) software.Simple frequency and percentages were the descriptive statistics used in this study for the analysis of results obtained. Chi-square was used to assess whether or not a relationship exists between risk factors and the prevalence of NTM at 95\% level of significance. The degree of association between the risk factors examined and NTM were analyzed using odds ratio (OR).

Results: From the 300 questionnaires analyzed, there was no significant statistical associated between demographic characteristics examined ( $p<0.05)$. However, female participants were higher $(56.3 \%)$ than the male (43.7\%). Participants within the age range of 35-44 had the higher positive cases $(20.8 \%)$. Participants who were married, had medium economic status and were civil servants had the higher NTM cases of $13.3 \%, 21.5 \%$ and $23.5 \%$ respectively. Among the risk factors examined, Cigarrete smoking, alcohol drinking and HIV status were significantly associated with the prevalence of NTM ( $p>0.05)$.

Conclusion: This study concludes that Cigarrete smoking, alcohol consumption and HIV status were the risk factors significantly associated with the prevalence of NTM within the study population. Hence, there is need for awareness creation and sensitization to enhance effective prevention and control of this public health threat.
\end{abstract}

Keywords: Demographic, Risk Factors, Tuberculosis, Non-tuberculous Mycobacteria, Kaduna State.

\section{Introduction}

Non-tuberculousmycobacteria (NTM) refer to all Mycobacterium species other than the pathogens of the M.tuberculosis complex (MTBC), M. leprae and $M$. ulcerans rarely cause disease in humans. Though, they may cause disease in 
individuals with history of lung disease or immunodeficiency, while some species may cause disease in elderly women without underlying pulmonary disease or immunodeficiency (Field, et al.,2004; Jamison, 2006; Aliyu, 2015). Of all the diseases caused by NTM, pulmonary disease is the most recurrent, followed by lymphadenitis in children, skin disease caused by Mycobacterium marinum (especially common in fish tank owners) and other extra-pulmonary or disseminated infections (Griffith, 2007; Aksamit et al., 2013; Van Ingen, 2013).

Pulmonary NTM are commonly found in the environment and can be isolated from sources such as water, soil, milk, dust, animals and birds (Jamison, 2006). Over 140 species of NTM have been reported in literature with the majority of these species rarely being isolated in clinical samples (Van Ingen, 2013). Studies have reported Mycobacterium avium complex (MAC) comprising the clinically important members $M$. intracellular, M. avium and M.chimaera are the most common. Other potentially pathogenic NTM species include $M$. chelonae, M. kansasii, M. xenopi, M. marinum, M. abscessus and $M$. fortuitum (Falkinham, 1996; Johnson et al., 2014). The occurrence of pulmonary disease from nontuberculous mycobacterium (NTM) is on the rise in Europe, North America, Asia and Southern Africa (Marras et al., 2015). However, studies conducted as far back as the late 1950s and early 1960s using traditional tools for identifying mycobacterial groups based on certain characteristics like speed of growth and morphology, have reported the isolation of NTM from both tuberculosis patients and the general public in some African countries including Nigeria (Zykov and Roulet, 1967). Pokam et al. (2012) report in Nigeria that $12.4 \% \quad(n=97)$ of AFB positive patients receiving treatment for pulmonary tuberculosis (TB) had infections with organisms other than mycobacteria while $4.1 \%$ (n=97) had NTM infections.

Researchers have reported globally that NTM infections are increasing and the spectrum of the
NTM diseases continue to broaden in most parts of the world (Buijtels., 2009; Chakraborty, 2010; Peyrani et al., 2014), the true incidence and prevalence of the infections is unknown (Jarzembowski et al., 2008; Chakraborty, 2010). The determination of incidence and prevalence was difficult due to the fact that NTM distribution is not fully known and infections are not reportable (Buijtels., 201; Peyrani et al, 2014).The lack of systematic reporting of NTM infection in most nations limits the ability to derive accurate estimates of incidence and prevalence of colonization infection (Buijtels, 2010).

Although, several demographic and risk factors have been implicated in association with NTB globally, the findings in this study will provide a data based information on the current status of NTM and associated risk factors in Kaduna State, Nigeria. This will help the policy makers in the formulation of policies for effective control and management of NTM in Nigeria. It will also increase awareness on certain factors that could predispose an individual to NTM infections among the study population. This study was aimed at assessing the demographic and risk factors associated with NTM among tuberculosis patientsin Kaduna State, Nigeria.

\section{Methods}

Study area: Kaduna state is located in the North West Geo-Political Zone of Nigeria, lying between attitudes $6^{\circ}$ and $11^{\circ}$ North and longitude $7^{\circ}$ and $44^{\circ}$ East and is 608 meters above sea level. The state wasthe former headquarters of the Northern Region Kaduna State occupies about $48,473.25$ sq.km, with a human population of over $6,066,562$ according to the census of 2006. Kaduna Statefalls within North-Western Nigeria. It has 23 Local Government Areas and three Senatorial Districts namely North (Kubau, Ikara, Makarfi, Soba, Sabon Gari, Zaria, Lere, Kudan), Central (Birnin Gwari, Giwa, Igabi, Kaduna North, Kaduna South, Chikun, Kajuru) and South (Jema'a, Jaba, Kaura, Zangon, Kaura, Kachia, Kagarko, Sanga). 
Study design: The study was a cross sectional study among TB patients attending Directly Observed Treatment Short Course (DOTS) clinics in the three (3) senatorial district of Kaduna State, Nigeria. Samples collected were from new smear positive TB patients attending the DOTS clinics in the selected hospitals (Ahmadu Bello University Teaching Hospital Shika; National TB Referral Hospital, Zaria and General Hospital, Kafanchan) (Fig. 1.).

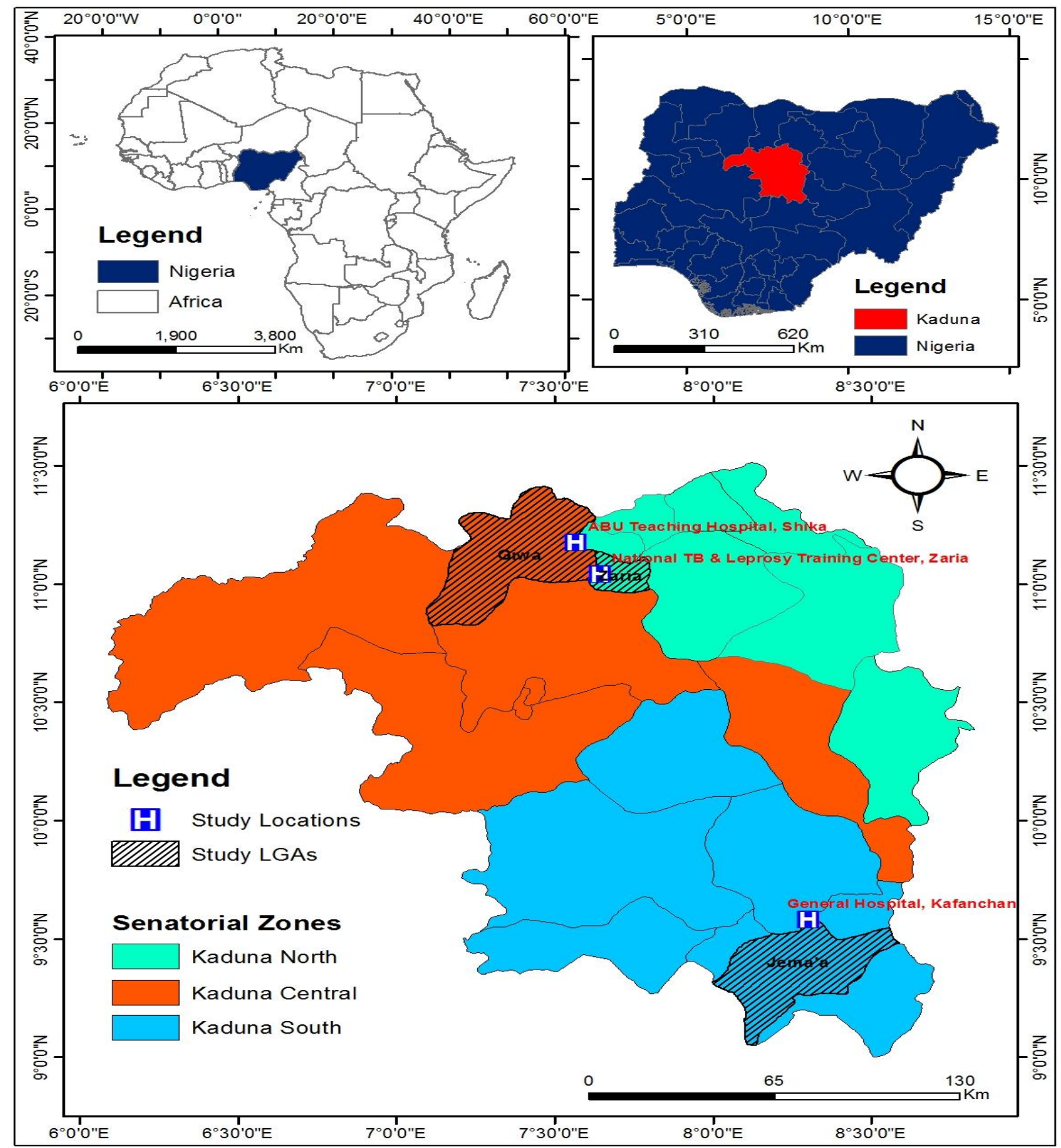

Figure 1: Map of Africa, Nigeria and Kakuna State showing the study locations

\section{Sample size determination}

Sample size was determined by the formula:

$\mathrm{N}=\underline{\mathrm{t}^{2} \mathrm{pq}}(\mathrm{WHO}, 2011)$.

$\mathrm{d}^{2}$

Where $\mathrm{N}=$ Minimum sample size $\mathrm{t}=$ standard normal distribution at $95 \%$ confidence interval (1.96)

$\mathrm{d}=$ allowable error taken as 0.05

$\mathrm{p}=$ known prevalence rate of the infection

$q=1-p$ 
$\mathrm{p}=$ known prevalence rate of NTM infection (15.5\%) (Aliyu et al., 2013)

$\mathrm{N}=\frac{\left(1.96^{2}\right) \times 0.1555 \times 0.845}{(0.05)^{2}}=201$

The calculated sample size was increased by $20 \%$ (WHO, 2003) giving a total sample size of 240 but because of the different locations, 300 samples were collected. A proportion to size (PPS) sampling as recommended by WHO, 2014 was used to allocate sample size across the selected hospitals.

Ethical approval: Ethical approval for this study was obtained from the State Ministries of Health, Kaduna State, Nigeria.

Inclusion Criteria: Smear positive TB cases, willingness to consent and the age of 15-years old and above.

Exclusion Criteria: Unwillingness to consent, smear negative TB cases and the age below 15years, Patients with confirmed Drug resistant TB, Patient confirmed to have TB using culture and clinically diagnosed pulmonary TB.

\section{Questionnaire Administration: Structured} questionnaire were used to collect demographic characteristics (age, sex, ethnicity, education, and marital status), health/behavioral factors (HIV, diabetes, smoking, alcohol use), environmental exposures (farming, animal contact, and dust season) and clinical variables (site, smear results).

\section{Results}

Socio-demographic characteristics and possible association with NTM is depicted in Table 1. It was observed that 22(13.0\%) out of 169 female participants were NTM positive while 17(13.0\%) of the male were positive for NTM, although the difference was not statistically significant ( $p$ $=0.992$ ). Patients with the age group of 35-44 had the highest NTM prevalence of $20.8 \%$ while the age between 25-34 had the lowest with $7.7 \%$, though, the difference was not significant statistically $(\mathrm{p}=0.111)$. More so, married participants had more NTM positive cases $(13.3 \%)$ than the single $(11.1 \%)$ and the difference was also not statistically significant $(\mathrm{p}=0.719)$. This study also revealed that participants with medium economic status had the highest NTM positive cases $(21.5 \%)$ while those with high economic status did not record positive cases of NTM $(0.0001 \%)$ and the association was not significant statistically $(\mathrm{p}=0.245)$. Furthermore, participants who were civil servants had the highest cases of NTM infection (23.5\%) and Livestock Keepers had the lowest $(9.4 \%)$ but the difference was not statistically significant ( $\mathrm{p}=$ 0.116).

The association between the examined risk factors and the prevalence of NTM among the study population is shown in Table 2. The findings in this study revealed a significant relationship between Cigarette smoking and NTM prevalence $(p=0.0001)$ and the smokers have three times higher chances of getting infected than the non Cigarrete smokers.

Furthermore, there was statistically significant relationship between alcohol drinking and NTM prevalence $(p=0.0001)$. Also, a significant relationship was established $(\mathrm{p}=0.0001)$ between HIV status and NTM infection in this study. More so, having diabetes was not statistically associated $(\mathrm{p}=0.106)$ with NTM prevalence in this study. This study also revealed that there was no significant relationship $(p=0.0694)$ between history of TB in the family and the prevalence of NTM. The association between being ever diagnosed of TB and the prevalence of NTM was also not statistically significant $(\mathrm{p}=0.066)$. It was also discovered in this study that dry season recorded the highest number of NTM cases than rainy season and the association was significant statistically $(\mathrm{p}=0.031)$. 
Table 1: Relationship between Sociodemographic factors and Nontuberculous Mycobacteria among the study participants

\begin{tabular}{|c|c|c|c|c|c|c|}
\hline $\begin{array}{l}\text { Socio-Demographic } \\
\text { Factors }\end{array}$ & $\begin{array}{c}\text { No of } \\
\text { Participants }\end{array}$ & No. Positive (\%) & Odds (C.I.) & $\mathbf{X}^{2}$ & Df & P-value \\
\hline \multicolumn{7}{|l|}{ Sex } \\
\hline Male & 131 & $17(13.0)$ & $0.998(0.681-1.463)$ & 0.00 & 1 & 0.992 \\
\hline Female & 169 & $22(13.0)$ & $1.002(0.745-1.346)$ & & & \\
\hline Total & 300 & 39(13.0) & & & & \\
\hline \multicolumn{7}{|l|}{ Age Group (years) } \\
\hline $15-24$ & 24 & $5(20.8)$ & & 8.958 & 5 & 0.111 \\
\hline $25-34$ & 39 & $3(7.7)$ & & & & \\
\hline $35-44$ & 53 & $11(20.8)$ & & & & \\
\hline $45-54$ & 56 & $5(8.9)$ & & & & \\
\hline $55-64$ & 58 & $10(17.2)$ & & & & \\
\hline$>65$ & 70 & $5(7.1)$ & & & & \\
\hline Total & 300 & 39(13.0) & & & & \\
\hline \multicolumn{7}{|l|}{ Marital Status } \\
\hline Married & 264 & $35(13.3)$ & $1.023(0.911-1.148)$ & 0.129 & 1 & 0.719 \\
\hline Single & 36 & $4(11.1)$ & $0.837(0.313-2.237)$ & & & \\
\hline Total & 300 & 39(13.0) & & & & \\
\hline \multicolumn{7}{|l|}{ Economic Status } \\
\hline High & 6 & $0(0.0)$ & & 2.812 & 2 & 0.245 \\
\hline Medium & 65 & $14(21.5)$ & & & & \\
\hline Low & 190 & $25(13.2)$ & & & & \\
\hline Total & 300 & 39(13.0) & & & & \\
\hline \multicolumn{7}{|l|}{ Occupation } \\
\hline Civil Servant & 34 & $8(23.5)$ & & 3.314 & 2 & 0.116 \\
\hline Farming & 181 & $23(12.7)$ & & & & \\
\hline Keeping Livestock & 85 & $8(9.4)$ & & & & \\
\hline
\end{tabular}

Table 2: Risk factors associated with Nontuberculous Mycobacteria among the study participants

\begin{tabular}{|c|c|c|c|c|c|c|}
\hline Risk Factors & $\begin{array}{c}\text { No of } \\
\text { Participants } \\
\end{array}$ & $\begin{array}{c}\text { No. positive } \\
(\%)\end{array}$ & Odds (C.I.) & $\mathbf{X}^{2}$ & df & P-value \\
\hline \multicolumn{7}{|c|}{ Cigarrete Smoking } \\
\hline Yes & 63 & $21(33.3)$ & $3.346(2.240-4.999)$ & 29.152 & 1 & $0.001 *$ \\
\hline No & 237 & 18(7.6) & $0.550(0.390-0.775)$ & & & \\
\hline Total & 300 & 39(13.0) & & & & \\
\hline \multicolumn{7}{|l|}{ Alcohol Drinking } \\
\hline Yes & 53 & $11(20.8)$ & $1.753(0.989-3.106)$ & 12.423 & 1 & $0.001 *$ \\
\hline No & 247 & $28(11.3)$ & $0.856(0.698-1.049)$ & & & \\
\hline Total & 300 & 39(3.0) & & & & \\
\hline \multicolumn{7}{|l|}{ HIV Status } \\
\hline Positive & 66 & $17(25.8)$ & & 13.429 & 2 & $0.001^{*}$ \\
\hline Negative & 219 & $22(10.0)$ & & & & \\
\hline Status not known & 15 & $0(0.0)$ & & & & \\
\hline Total & 300 & 39(13.0) & & & & \\
\hline \multicolumn{7}{|c|}{ Having Diabetes Mellitus } \\
\hline Yes & 15 & $4(26.7)$ & $2.434(0.815-7.266)$ & 2.608 & 1 & 0.106 \\
\hline No & 285 & $35(12.3)$ & $0.937(0.840-1.045)$ & & & \\
\hline Total & 300 & 39(13.0) & & & & \\
\hline \multicolumn{7}{|c|}{ History of TB in family } \\
\hline Yes & 11 & $1(9.1)$ & $0.669(0.88-5.085)$ & 0.154 & 1 & 0.694 \\
\hline No & 289 & $38(13.1)$ & $1.013(0.958-1.072)$ & & & \\
\hline Total & 300 & 39(13.0) & & & & \\
\hline \multicolumn{7}{|c|}{ Being Ever Diagnosed of TB } \\
\hline Yes & 9 & $3(33.3)$ & $3.346(0.872-12.836)$ & 3.392 & 1 & 0.066 \\
\hline No & 291 & $36(12.4)$ & $0.945(0.861-1.036)$ & & & \\
\hline Total & 300 & $39(13.0)$ & & & & \\
\hline \multicolumn{7}{|c|}{ Season of Diagnosis } \\
\hline Dry Season & 162 & $22(14.0)$ & & 0.14 & 2 & $0.031^{*}$ \\
\hline Rainy Season & 138 & $17(12.3)$ & & & & \\
\hline Total & 300 & 39(13.0) & & & & \\
\hline
\end{tabular}




\section{Discussions}

Studies have supported the strong effects of demographic factors such as gender, age, marital status, economic status and occupation on the incidence of NTM globally. In this study, female participants recorded the higher cases of NTM occurrence, although the association was not significant statistically. This might not be unconnected with the fact that women have more psycho-social consequences of stigmatizing ailments and consequently avoid presenting themselves to health facilities timely. This finding agreed with the report of Thomas, 2010 who reported higher prevalence of NTM among women than men in Ibadan, Nigeria. Our findings are in line with the report of Falodun et al., (2016) who recorded higher NTM prevalence in women $(66.7 \%)$ than men $(33.3 \%)$ in Ibadan, Nigeria.This findings also agrees with the report of Cassidy et al., (2009) in Oregon, USA that had strong association of NTM infection in women than men. In this study, the risk factors that showed significant association with the prevalence of NTM were Cigarrete smoking, alcohol drinking , HIV status and season of diagnosis. Studies have established that Cigarrete smoking is associated with the infections of the lungs which could predispose a smoker to TB infections of all forms. HIV and TB coinfections has been a serious health challenges globally and thus, those who are HIV positive have the higher risk of NTM infections which might be due to decreased immunity. Our findings agrees with the reports of Ghio et al., (1990); Halmilton et al., (2008); Pasula et al., (2009) and Aliyu et al. (2013) who obtained similar results.

We also observed a significant relationship between NTM prevalence and season. NTM was higher during the Harmattan month of the dry season and decreases during the rainy season. Harmattan is a West African trade wind that occurs during the winter which is characterized with heavy dust which could increase the rate of NTM infections in exposed individuals. This findings agrees with the report of Aliyu et al.,
(2013) who recorded higher prevalence of NTM during Harmattan season in Kaduna State, Nigeria. Furthermore, the findings affirms the report of Corbett et al. (2000) in South Africa that a dust-exposed job at diagnosis was a risk factor for NTM lung disease.

\section{Conclusion}

The prevalence of NTM in strong association with risk factors such as Cigarrete smoking, alcohol consumption and HIV status among the participants portents great challenge to public health in Nigeria. Furthermore, the fact that NTM was higher among the active workforce poses a threat to the nation. Therefore, this call for serious attention and needs proper awareness creation for effective management and control of NTM burden in Nigeria.

\section{References}

1. Aksamit, T.R., Philley, J.V., Griffith, D.E. (2013). Non-tuberculous Mycobacterial (NTM) lung disease:The top ten essentials. Respiratory Medicine;108:417-425.

2. Aliyu, M.S. (2015). Prevalence of MultiDrug Resistant Mycobacterium Tuberculosis (MDR-TB) in Kaduna State, Nigeria. Ph.D. Thesis. Department of Microbiology, Ahmadu Bello University, Zaria-Nigeria. pp 1-104

3. Buijtels, P.C., van der Sande, M.A., de Graaff, C.S., Parkinson, S., Verbrugh, H.A., Petit, P.L.C., vanSoolingen, D.(2009). Non-tuberculous mycobacteria. Zambia Emergence of Infectious Disease; 15(2):24-242.

4. Cassidy PM, Hedberg K, Saulson A, McNelly E, Winthrop KL. (2009). Nontuberculous Mycobacterial Disease Prevalence and Risk Factors: A Changing Epidemiology Clinical Infectious Diseases; 49: e124-9 Chakraborty P. (2010). A text book of Microbiology. 1st ed. London: New Central Book Agency (P) Ltd. 
5. Falodun, I.O., Fagade, O. E. and Cadmus, S. I. B. (2016). Prevalence of Nontuberculous Mycobacteria Infections in Patients Diagnosed with Pulmonary Tuberculosis in Ibadan. International Journal of Tropical Disease \& Health 18(3): 1-8, 2016, Article no.IJTDH.28058 www.sciencedomain.org.

6. Falkinham, J.O. (1996). Epidemiology of infection by nontuberculous mycobacteria. ClinicalMicrobiology Reviews, 9 (2):177215.

7. Field SK, Fisher D, Cowie RL. Mycobacterium avium complex pulmonarydisease in patients without HIV infection. Chest. 2004; 126(2):566-81.

8. Ghio, A.J., Kennedy, T.P., Schapira, R.M., Crumbliss,A.L., Hoidal, J.R. (1990). Hypothesis: is lung disease after silicate inhalation caused by oxidant generation? Lancet 336: 967-969.

9. Griffith, D.E., Aksamit, T., Brown-Elliott, B.A., Catanzaro, A., Daley, C., Gordin, F. (2007). AnOfficial ATS/IDSA statement: diagnosis, treatment, and prevention of non-tuberculousMycobacterial diseases. Annual Journal of Respiratory and Critical Care Medicine,175:367-416.

10. Hamilton, R.F., Jr., Thakur, S.A., Holian, A. (2008). Silica binding and toxicity in alveolar macrophages. Free radical biology \& medicine 44: 1246-1258.

11. Jamison, D.T. (2006). Disease and Mortality in Sub-Saharan Africa. Washington: World BankPublications; 2006. 180.

12. Jarzembowski, J.A and Young, M.B. (2008). Review Article: Non-tuberculous Mycobacterial infections.Arch. Pathol. Lab. Med. 132:1333-1341.
13. Johnson, M.M., and Odell, J.A. (2014). Non-tuberculous Mycobacterial pulmonary infections. Journal of Thoracic Disease, 6(3):210-220.

14. Marras, T.K., Daley, C.L. (2015). Epidemiology of human pulmonary infection withnon-tuberculous mycobacteria Clinics in chest medicine, 23: 553-567.

15. Peyrani, P., Ramirez, J.A. (2014).Nontuberculous Mycobacterial pulmonary infections. In: Feldman, C., Polverino, E., Ramirez, J.A.,editors. Pulmonary Complications of HIV. Switzerland: European Respiratory Society, 66: 128

16. Pasula, R., Britigan, B.E., Turner, J., Martin, W.J. (2009). Airway delivery of silica increases susceptibility to mycobacterial infection in mice: potential role of repopulating macrophages. Journal of immunology 182: 7102-7109.

17. Pokam, B.T., Asuquo, A.E. (2012). Acidfast bacilli other than mycobacteria in tuberculosis patients receiving directly observed therapy short course in Cross River State, Nigeria. Tuberculosis Research and Treatment. 1-4.

18. World Health Organization. (2011). Global tuberculosis control. WHO/HTM/TB/2011.16. Available: http://whqlibdoc.who.int/publications/201 1/978241564380 eng.pdf

19. World Health Organization. (2013). Global Tuberculosis Report. Pages 159,162 \& 24. Available: http://www.who.int/tb/publications/global _report/en/. Accessed 5 May 2015.

20. Zykov, M.P., and Roulet, H. (1967). Nontuberculosis mycobacteria in Africa. 3. Formamidaseactivity-its evaluation and practical application. Bulletin of the World HealthOrganization,37: 947-951. 